\title{
Burst Shower Events Observed by the Telescope Array Surface Detector
}

\author{
Takeshi Okuda* \\ Ritsumeikan University \\ E-mail: okuda@icrr.u-tokyo.ac.jp \\ John Belz, William Hanlon \\ University of Utah
}

Masaki Fukushima, Kazumasa Kawata, Eiji Kido, Toshiyuki Nonaka, Hiroyuki Sagawa, Masahiro Takeda, Akimichi Taketa, Hisao Tokuno

University of Tokyo

\section{Nobuyuki Sakurai}

Nagoya University

for the Telescope Array Collaboration ${ }^{\dagger}$

The Telescope Array (TA) Surface particle Detector (TASD) has observed short time bursts of air-shower like events. We checked the correlation for time and position between these burst events and lightning data, and found evident correlation. Some features of the burst events are similar to the normal cosmic ray air-shower, and some are not. We report on the observed bursts of air-shower like events and their correlation with lightning.

The 34th International Cosmic Ray Conference,

30 July- 6 August, 2015

The Hague, The Netherlands

\footnotetext{
* Speaker.

${ }^{\dagger}$ Full author list : http://www.telescopearray.org/images/papers/ICRC2015-authorlist.pdf
} 


\section{Introduction}

There are some reports about the observation of energetic radiation from thunderclouds. Some of these reports discuss bursts of energetic radiation observed from space, known as terrestrial gamma-ray flashes[7][2]. Their mechanism is still unknown, but they are believed to be associated with upward lightning flashes at the top of thunderclouds. Other reports discuss the increase of arrival rates of single radiation along with thundercloud at ground. Finally, observations of energetic radiation have been associated with particular lightning processes in the flash.

The natural lightning flash consists of several processes, known as the stepped leader, return stroke, dart leader and subsequent return stroke. The leader direction may be up or down and of positive or negative polarity, hence there are four types of lightning. However, except for lightning strikes on tall objects, most lightning starts with negative charges moving downward.

Moore, et al[9] reported the observation of energetic radiation from stepped leaders, using $\mathrm{NaI}$ as a radiation detector. Dwyer, et al[4] reported the observation of energetic radiation from dart leader also using $\mathrm{NaI}$ as a radiation detector, for rocket triggered lightings. Dwyer, et al[5] reported the observation of energetic radiation from stepped leaders, and Dwyer, et al[6] reported radiation associated with the return stroke, for natural cloud-to-ground lightning.

The electric fields present in a typical thunderstorm are not sufficient to start the stepped leader process, the beginning of lightning. Therefore, it has been hypothesized that the atmospheric ionization caused by cosmic ray air-showers plays a role in triggering lightning. From this side, there are some reports about energetic radiation with lightning. Gurevich, et al[8] reported the coincidence of air-showers with lightning, using $\mathrm{NaI}$ and gas-counter as radiation detectors.

The detectors used in these prior observations were mainly NaI, and sometimes gas-counters. TASD uses plastic scintillator of $3 \mathrm{~m}^{2}$ area, which is approximately 300 times the area of the $\mathrm{NaI}$ detectors but cannot measure the energy of individual particles. TASD responds 10 times faster than NaI detectors. Prior particle detectors were deployed over several square kilometers. TASD coverage is about 300 times larger, although it is inefficient for small air-showers due to the lower number density of the detectors.

\section{Observed Burst Events}

The Telescope Array (TA) experiment, located in Midwest Utah, USA, consists of two types of detector (Figure 1). Both detection methods observe the high energy phenomena known as "air-showers", which are generated by ultra high energy cosmic rays. One type of TA instrument is the atmospheric fluorescence telescope, and the other is an array of ground surface particle detectors. In contrast to the atmospheric fluorescence which is observable only on moonless nights, the TA Surface Detector (TASD) runs 24 hours per day, throughout the year. TASD consists of 507 individual particle detectors. The particle-sensitive part of a TASD detector element is shown in Figure 1. The TASD elements are deployed on a $1.2 \mathrm{~km}$ square grid, covering a total of $680 \mathrm{~km}^{2}$. When three adjacent elements detect a signal corresponding to three or more particles within an $8 \mu$ s window, waveforms of all detectors within $\pm 32 \mu$ s are recorded[1]. TASD is designed to detect all air showers from cosmic rays with energy greater that $10 \mathrm{EeV}\left(=10^{19} \mathrm{eV}\right)$. TASD data used for this report was collected between May 11, 2008 and May 4, 2013. 

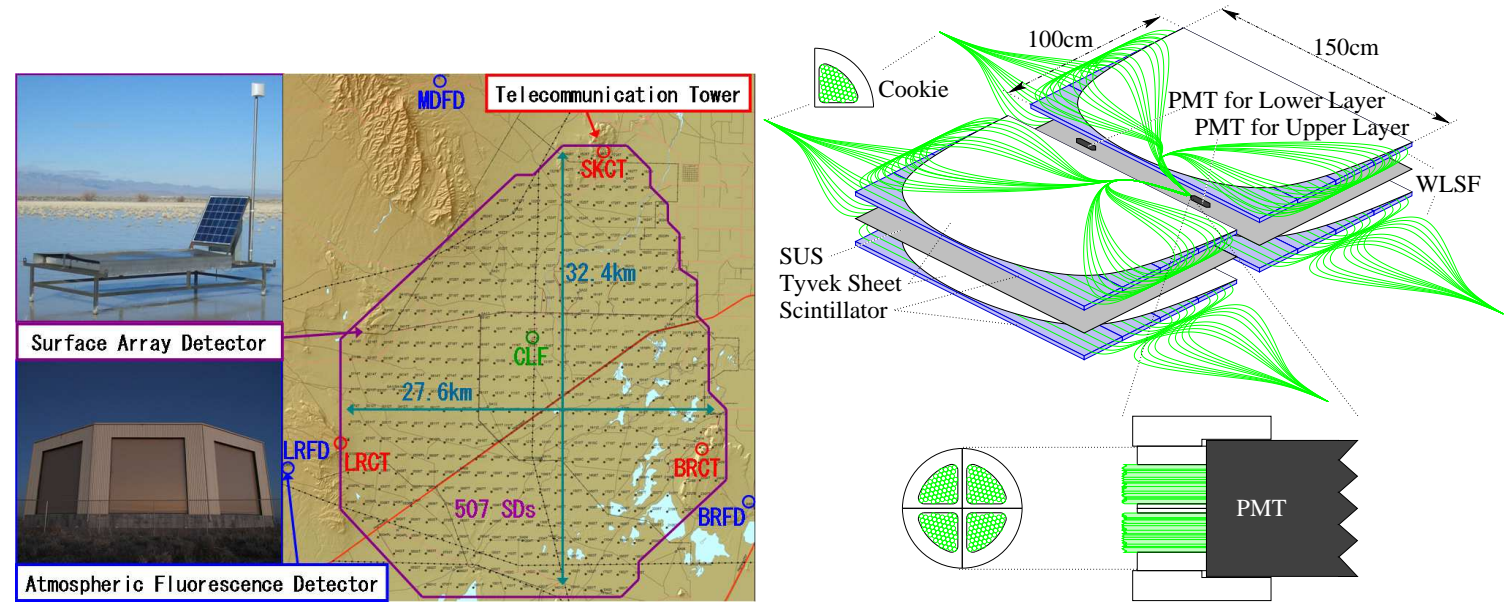

Figure 1: Telescope Array Experiment. Left: 507 ground surface particle detectors (tiny dots above) are surrounded by 3 atmospheric fluorescence telescope stations. Right: Particle detecting part of TASD consists of 2 layers of plastic scintillators. Each scintillation layer has $1.2 \mathrm{~cm}$ thickness and provides information respectively via wavelength-shifting fibers.

We searched for bursts of air-shower events. The criteria used in this burst search was more than three events within 1 ms. 10 such bursts were found. The expectation for such bursts due to chance coincidence of single shower events is less than $10^{-4}$ for a five-year observation period. In addition, although bursts were not selected by position, the shower cores of each burst are found to be localized within a $\sim 1 \mathrm{~km}$ radius. Considering the event which could not generate shower trigger but are found in waveforms, the time gaps of events in a burst are distributed from several to a hundred microseconds. Five of the ten bursts contained air-showers which are reconstructible by minor modification to the cosmic ray air-shower reconstruction programs, in which unreconstructible early waveforms are artificially removed.

In this reconstruction, the air-shower front is modeled as a sphere expanding at the speed of light from a single point in the sky, and the altitude of this point is a parameter of a fit to the shower. The reconstructed air-shower front curvature is much greater for the burst events than for usual cosmic ray air-showers. Therefore, the altitudes of the reconstructed shower origin for the burst events are at an atmospheric depth much deeper than those of usual cosmic ray air-showers. In addition, the reconstructed air-shower directions for each burst tend to point small region at low sky. The waveforms captured in the nearest shower core scintillator detectors do not have a sharp rising edge for many of the burst events, and in this aspects the waveforms are in contrast to those of normal cosmic ray air-showers. However, time-integrated energy deposit in the TASD scintillators is similar to that of cosmic ray air showers.

\section{Correlation With Lightning}

We checked these bursts for correlation with the Vaisala lightning database. This database comes from the U.S. National Lightning Detection Network (NLDN). The NLDN detects lightning by multi-position very low-frequency antennas, and derives lightning information by radio arrival timing and waveform[3][10]. This lightning data list contains time, position, peak current and a 

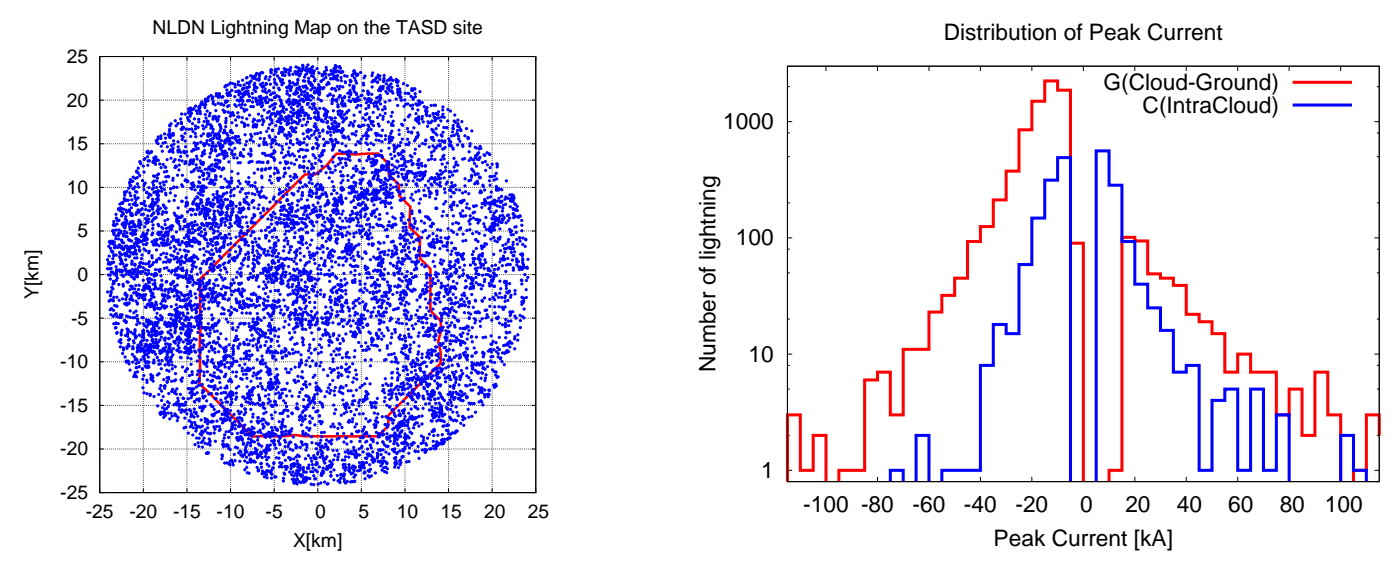

Figure 2: NLDN data distribution. Left: Lightning map on the TA site. Blue points show individual strokes. Red line shows the area of TASD. Right: Absolute peak current distribution for 4 categories which combined with peak current polarity and flag which indicates intracloud or cloud-ground lightning.

$\mathrm{C} / \mathrm{G}$ flag indicating whether the lightning is intracloud or cloud-to-ground lightning. NLDN is somewhat inefficient for intracloud lightning.

The lightning list used in this report was selected by position within 15 miles from the center of the TA site which covers the whole TASD area, and also by time between May 2008 and April 2013. $79 \%$ of the listed lightning is flagged as cloud-to-ground lightning. $85 \%$ of the listed lightning has negative peak current. The location and peak current distribution of lightning on the list is shown in Figure 2.

We checked the correlation between the five reconstructed bursts and lightning by timing. We categorize this search by two types of correlation, which we call "synchronized" and "related".

The criteria distinguishing those events which are "synchronized" with lightning is that the time difference between burst and lightning is less than $1 \mathrm{~ms}$. Four of the five reconstructed bursts have synchronized lightning. Although no requirement is placed on position, all synchronized lightning is located in the vicinity of burst air-shower events. All synchronized lightning is flagged by NLDN as intracloud lightning having negative peak current. Considering the intracloud lightning detection efficiency of the NLDN, the observed air-shower bursts and lightning are well-synchronized.

The criteria distinguishing events which are "related" to lightning is that the time difference between burst and lightning is less than $200 \mathrm{~ms}$, excluding synchronized events. Three bursts in the four above have related lightning. There is no selection by position, but all related lightning is in the vicinity of burst air-shower events. All related lightning is flagged by NLDN as cloud-to-ground lightning and negative peak current. These related lightning strikes are subsequent components of the synchronized lightning events.

Our check of correlation between shower bursts and lightning shows that the observed bursts are clearly synchronized with negative intracloud lightning. The absolute peak current of the synchronized lightning is extremely high compared with other NLDN activity in the vicinity of the TASD. Therefore, these bursts are very rare phenomena.

Of the five bursts which are not reconstructible, two are correlated in time and position with 
lightning. One of the five reconstructed bursts and three of the five unreconstructible bursts are not correlated with lightning by the above definitions. However, there are multiple lightning strikes near the times of the observed bursts, therefore all ten bursts observed in five years occur during thunderstorms.

\section{Conclusion and Discussion}

We have detected bursts of high energy events using the TASD, which seem to come from negative high-current intracloud lightning as judged by correlation of time and position. There is no evidence that the bursts come from cloud-to-ground lightning.

What generates the individual events within a burst? We do not have a clear answer. If these are interpreted as arising from cosmic rays, the trigger rate in a burst is inconsistent with the observed energies. We summarize the features of the bursts as follows:

1. This burst phenomenon does not arise from thunderstorms at random, but rather comes from negative high current intracloud lightning.

2. The reconstructed shower directions for events within a burst, within reconstruction accuracy, indicate that they arise from a very small region at low altitude.

3. The showers seem to start their development low in the atmosphere compared to cosmic rays, as determined by the shower front curvature.

4. The waveforms exhibit time gaps in the detected radiation which is consistent to that expected by the stepped leader process (several tens of microseconds).

5. The leading edge of the waveforms at the shower core are less-sharply rising than those of typical cosmic ray air-showers, for many events.

To prior observations of energetic radiation associated with lightning, we add somewhat unique information especially such as spatial distribution, derived from air-shower reconstruction using a faster waveform from a larger detector for higher current intracloud lightning.

\section{Acknowledgements}

The Telescope Array experiment is supported by the Japan Society for the Promotion of Science through Grants-in-Aid for Scientific Research on Specially Promoted Research (21000002) "Extreme Phenomena in the Universe Explored by Highest Energy Cosmic Rays" and for Scientific Research (19104006), and the Inter-University Research Program of the Institute for Cosmic Ray Research; by the U.S. National Science Foundation awards PHY-0307098, PHY-0601915, PHY-0649681, PHY-0703893, PHY-0758342, PHY-0848320, PHY-1069280, PHY-1069286, PHY1404495 and PHY-1404502; by the National Research Foundation of Korea (2007-0093860, R3210130, 2012R1A1A2008381, 2013004883); by the Russian Academy of Sciences, RFBR grants 11-02-01528a and 13-02-01311a (INR), IISN project No. 4.4502.13, and Belgian Science Policy under IUAP VII/37 (ULB). The foundations of Dr. Ezekiel R. and Edna Wattis Dumke, Willard L. 
Eccles, and George S. and Dolores Doré Eccles all helped with generous donations. The State of Utah supported the project through its Economic Development Board, and the University of Utah through the Office of the Vice President for Research. The experimental site became available through the cooperation of the Utah School and Institutional Trust Lands Administration (SITLA), U.S. Bureau of Land Management, and the U.S. Air Force. We also wish to thank the people and the officials of Millard County, Utah for their steadfast and warm support. We gratefully acknowledge the contributions from the technical staffs of our home institutions. An allocation of computer time from the Center for High Performance Computing at the University of Utah is gratefully acknowledged.

The lightning data on this paper was served from Vaisala Inc. We appreciate Vaisala's academic research policy. And my work for lightning with TASD is supported by Mitsubishi Foundation.

\section{References}

[1] T.AbuZayyad, et al, NIM Phys. Res. A (2012), 689, p87-97

[2] M.S.Briggs, et al, J. Geophys. Res. (2010), vol 115, A07323

[3] K.L.Cummins and M.J.Murphy, IEEE Trans. (2009), vol 51(3), p499-518

[4] J.R.Dwyer, et al, Science (2003), 299, p694-697

[5] J.R.Dwyer, et al, Geophys. Res. Rett. (2005), vol 32, L01803

[6] J.R.Dwyer, et al, J. Geophys. Res. (2012), vol 117, A10303

[7] G.J.Fishman, et al, Science (1994), 264, p1313-1316

[8] A.V.Gurevich, et al, Phys. Rett. A (2009), 373, p3550-3553

[9] C.B.Moore, et al, Geophys. Res. Rett. (2001), vol 28, p2141-2144

[10] A.Nag, et al, J. Geophys. Res. (2011), vol 116, D02123 